\title{
Fighting Uncertainty with Uncertainty: A Baby Step
}

\author{
Ravi Kashyap \\ City University of Hong Kong, Hong Kong, China \\ Email: ravi.kashyap@stern.nyu.edu
}

How to cite this paper: Kashyap, R. (2017) Fighting Uncertainty with Uncertainty: A Baby Step. Theoretical Economics Letters, 7, 1431-1452.

https://doi.org/10.4236/tel.2017.75097

Received: July 6, 2017

Accepted: August 11, 2017

Published: August 14, 2017

Copyright (C) 2017 by author and Scientific Research Publishing Inc. This work is licensed under the Creative Commons Attribution International License (CC BY 4.0).

http://creativecommons.org/licenses/by/4.0/

\begin{abstract}
We can overcome uncertainty with uncertainty. Using randomness in our choices and in what we control, and hence in the decision making process, we could potentially offset the uncertainty inherent in the environment and yield better outcomes. The example we develop in greater detail is the news-vendor inventory management problem with demand uncertainty. We briefly discuss areas, where such an approach might be helpful, with the common prescription, “Don't Simply Optimize, Also Randomize; perhaps best described by the term-Randoptimization". 1) News-Vendor Inventory Management; 2) School Admissions; 3) Journal Submissions; 4) Job Candidate Selection; 5) Stock Picking; 6) Monetary Policy. This methodology is suitable for the social sciences since the primary source of uncertainty is the members of the system themselves and presently, no methods are known to fully determine the outcomes in such an environment, which perhaps would require being able to read the minds of everyone involved and to anticipate their actions continuously. Admittedly, we are not qualified to recommend whether such an approach is conducive for the natural sciences, unless perhaps, bounds can be established on the levels of uncertainty in a system and it is shown conclusively that a better understanding of the system and hence improved decision making will not alter the outcomes.
\end{abstract}

\section{Keywords}

Fight, Uncertainty, Social Science, Natural Science, Baby Step, Randoptimization

\section{Introduction}

\subsection{True Comparison Theory or Lack Thereof}

Keeney [1] presents an overview of decision analysis: what it is, what it can and cannot do, and how to do it, including a summary of the axioms, while main- 
taining that "complexity cannot be avoided in making decisions". He defines decision analysis informally as "a formalization of common sense for decision problems which are too complex for informal use of common sense"; and more technically as "a philosophy, articulated by a set of logical axioms, and a methodology and collection of systematic procedures, based upon those axioms, for responsibly analyzing the complexities inherent in decision problems." Pratt, Raiffa \& Schlaifer [2] is a detailed discussion of the axioms of decision analysis; Raiffa [3] is an interesting personal account. The similarities of this approach to subjective or personal evaluation of probabilities to formulate decisions under uncertainty (Ramsey [4]; Savage [5]) are to be noted.

If we admit that common sense cannot deal with complexity, especially when subjective decisions from the participants themselves are creating complexity; we need to be open to the possibility that perhaps, extremely careful analysis leading to improved decision making might merely be an illusion. A hall mark of the social sciences is the lack of objectivity. Here we assert that objectivity is with respect to comparisons done by different participants and that a comparison is a precursor to a decision (Kashyap [6]).

Conjecture 1. Despite the several advances in the social sciences, we have yet to discover an objective measuring stick for comparison, a so called, True Comparison Theory, which can be an aid for arriving at objective decisions.

The search for such a theory could again be compared, to the medieval alchemists' obsession with turning everything into gold (Kashyap [7]). For our present purposes, the lack of such an objective measure means that the difference in comparisons, as assessed by different participants, can effect different decisions under the same set of circumstances. Hence, despite all the uncertainty in the social sciences, the one thing we can be almost certain about is the subjectivity in all decision making.

This lack of an objective measure for comparisons, makes people react at varying degrees and at varying speeds, as they make their subjective decisions. A decision gives rise to an action and subjectivity in the comparison means differing decisions and hence unpredictable actions. This inability to make consistent predictions in the social sciences explains the growing trend towards comprehending better and deciphering the decision process and the subsequent actions, by collecting more information across the entire cycle of comparisons, decisions and actions. Another feature of the social sciences is that the actions of participants affects the state of the system, effecting a state transfer which perpetuates another merry-go-round of comparisons, decisions and actions from the participants involved. This means, more the participants, more the changes to the system, more the actions and more the information that is generated to be gathered.

\subsection{The Uncertainty Principle of the Social Sciences}

Lawson [8] argues that the Keynesian view on uncertainty (that it is generally 
impossible, even in probabilistic terms, to evaluate the future outcomes of all possible current actions: (Keynes [9] [10] [11]), far from being innocuous or destructive of economic analysis in general, can give rise to research programs incorporating, amongst other things, a view of rational behavior under uncertainty, which could be potentially fruitful. Knight [12] in his seminal work, "Risk, Uncertainty and Profit", makes a distinction between Uncertainty and Risk that can be immensely enlightening in our efforts to comprehend uncertainty.

First, we note a difference between the Keynesian and Knightian views of uncertainty (Hoogduin [13]): Knight mainly focuses on the distinction between numerically measurable and not numerically measurable probabilities; Keynes stresses the slight amount of knowledge on which probabilities often have to be based. There are many definitions of uncertainty; for simplicity and to suit our present purpose, we consider the following definition based on the Keynesian and Knightian views:

Definition 1. Uncertainty is the lack of certainty; a situation of having limited knowledge where it is impossible to exactly describe the existing state, a future outcome, or even be aware of all the possible outcomes. Risk, means, in some cases a quantity susceptible of measurement, while at other times it is something distinctly not of this character. Uncertainty then becomes risk that is immeasurable and not possible to calculate. ${ }^{1,2}$

McManus and Hastings [14] clarify the wide range of uncertainties that affect complex engineering systems and present a framework to understand the risks (and opportunities) they create and the strategies system designers can use to mitigate or take advantage of them. These viewpoints hold many lessons for policy designers in the social sciences and could be instructive for researchers looking at ways to understand and contend with complex systems, keeping in mind the caveats of dynamic social systems. Kashyap [7] discusses the uncertainty principle of the social sciences and the use of a feedback loop to overcome the phenomenon, wherein participants observe the results and change their actions that could potentially lead to unexpected and different consequences.

Conjecture 2. The Uncertainty Principle of the Social Sciences can be stated as, "Any generalization in the social sciences cannot be both popular

${ }^{1}$ With this definition of risk and uncertainty, our paper must perhaps be titled: Fighting Uncertainty with Risk. Overlooking the possibility that the present title is more catchy, we note that our attempts at controlled or measurable uncertainty might lead to unforeseen consequences, which might not be easily measured. In general, even when we believe that we have a good idea of the risks, we might be over estimating our ability to estimate the risks (the effects of our actions on any social system and the outcomes therein), which is precisely the definition of uncertainty we have used.

${ }^{2}$ To provide readers a taste of the many flavors of commonly used definitions, we note that: Risk is also the potential of gaining or losing something of value (Kungwani [57]). Values (such as physical health, social status, emotional well-being, or financial wealth) can be gained or lost when taking risk, resulting from a given action or inaction, foreseen or unforeseen. Risk can also be defined as the intentional interaction with uncertainty. Risk can be seen as a state of uncertainty where some possible outcomes have an undesired effect or significant loss. Measurement of risk involves coming up with a set of measured uncertainties where some possible outcomes are losses, and factoring in the magnitudes of those losses. Measurement of uncertainty involves an effort to assess as best as feasible, a set of possible states or outcomes where probabilities are assigned to each possible state or outcome. 
and continue to yield predictions, or in other words, the more popular a particular generalization, the less accurate will be the predictions it yields".

This is because as soon as any generalization and its set of conditions becomes common knowledge, the entry of many participants shifts the equilibrium or the dynamics, such that the generalization no longer applies to the known set of conditions. An observation is likely to be more popular when there are more people comprising that system; and it is important to try and explicitly understand, where possible, how predictions can go awry. Every social system then operates under the overarching reach of this principle.

This varying behavior of participants in a social system will give rise to unintended consequences (Kashyap [15] [16]) and as long as participants are free to observe the results and modify their actions, this effect will persist ${ }^{3}$. (Simon [17]) points out that any attempt to seek properties common to many sorts of complex systems (physical, biological or social), would lead to a theory of hierarchy, since a large proportion of complex systems observed in nature exhibit hierarchic structure.

Kashyap [18] [19] consider ways to reduce the complexity of social systems, which could be one way to mitigate the effect of unintended outcomes. While attempts at designing less complex systems are worthy endeavors, reduced complexity might be hard to accomplish in certain instances and despite successfully reducing complexity, alternate techniques at dealing with uncertainty are commendable complementary pursuits. It might be possible to observe historical trends (or other attributes) and make comparisons across fewer number of entities; though in large systems where there are numerous components or contributing elements, this can be a daunting task and constructing measures across groups or aggregations of smaller systems need to be explored (Kashyap [6] [20]).

\subsection{Randoptimization}

Kleywegt \& Shapiro [21] is a detailed account of decision making under uncertainty and how decision problems are often formulated as optimization problems and solved using stochastic optimization techniques. In this present paper, we take a step further and look at ways in which introducing randomness in our choices and in what we control, and hence in the decision making process, could potentially offset the uncertainty inherent in the environment and yield better outcomes.

In short we try to overcome uncertainty with uncertainty. Such an approach, while seemingly absurd, is the ideal medicine for the even greater absurdity in the decision making process that has become prevalent in today's society. It takes care of the issues that crop up due to the limitations in our understanding

${ }^{3}$ Sergei Bubka http://en.wikipedia.org/wiki/Sergey_BubkaSergey

Bubka: http://en.wikipedia.org/wiki/Sergey_Bubka] is our Icon of Uncertainty. As a refresher for the younger generation, he broke the pole vault world record 35 times. We can think of regulatory change or the utilization of newer methods and techniques as raising the bar. Each time the bar is raised, the spirit of Sergei Bubka, in all of us, will find a way over it. 
of complex systems and the widely acknowledged problem that most of our measurements are highly prone to errors.

Remark 1. Necessity is the mother of all creation (invention or innovation), but the often forgotten father, is frustration.

Pondering on the sources of uncertainty and the tools we have to capture it, might lead us to believe that, either, the level of our mathematical knowledge is not advanced enough, or, we are using the wrong methods. Many interesting situations in life (See Section 3), are caused by the uncertainty inherent in them, which we (all researchers and society) seem to be looking to solve using logic (mathematics). This paper is meant to illustrate why perhaps dealing with uncertainty, something twisted, requires an equally twisted approach and perhaps, solutions might not be obtained using straight techniques that rely on precision. The dichotomy between logic and randomness is a topic worth pursuing on many fronts. Our innovation is one possible alternative methodology, succinctly expressed as, "Fighting Uncertainty with Uncertainty".

This technique is suitable for the social sciences since the primary source of uncertainty are the members of the system themselves and presently, no methods are known to fully determine the outcomes in such an environment, which perhaps, would require being able to read the minds of everyone involved and to anticipate their actions continuously. Admittedly, we are not qualified to recommend whether such an approach is conducive for the natural sciences, unless perhaps, bounds can be established on the levels of uncertainty in a system and it is shown that a better understanding of the system and hence improved decision making will not alter the outcomes. Barring such a bound on the level of uncertainty it is advisable to understand the sources that cause arbitrary outcomes and follow more traditional methods.

The central innovation can be understood as optimizing to get an interval of satisfactory performance and randomizing over that interval. The goal is not just to optimize, but to identify a region of acceptable performance and set the parameters of a probability distribution over that region, and sample from it to provide an agreeable level of performance. Agents looking to optimize will randomize over an optimal region, hence this approach is called "Randoptimization". Due to measurement errors and other uncertainty, we can never be certain of any optimization we perform, rather it is better to randomize over acceptable states.

The example we develop in greater detail is the news-vendor inventory management problem with demand uncertainty. The proposition and theorem are new results and they depend on existing results which are given as Lemmas. We briefly discuss areas, where such an approach might be helpful, with the common prescription, "Don't Simply Optimize, Also Randomize; perhaps best described by the term-Randoptimization".

1) News-Vendor Inventory Management Problem (Section 2);

2) School Admissions;

3) Journal Submissions; 
4) Job Candidate Selection;

5) Stock Picking.

6) Monetary Policy.

\section{News-Vendor Problem}

The first of our case studies delves into the classical and well studied problem in the supply chain management literature and is purely about inventory control. We start with a basic model (Arrow, Harris \& Marschak [22]; Chen and Federgruen [23]; Cachon \& Lariviere [24]) and introduce the notion of random order quantity under different levels of uncertainty. (Khouja [25]; Qin, Wang, Vakharia, Chen \& Seref [26]) provide a comprehensive review and suggestions for future research. Interesting extensions include (Gallego \& Moon [27]), situations where the distribution is unknown but the mean and variance are known; (Gallego \& Van Ryzin [28]), changing the price dynamically; (Wu, Li, Wang \& Cheng [29]) stockout costs under a mean variance framework; (Wilson \& Sorochuk [30]), revenues from a secondary stream related to the product being sold are factored in; (Rubio-Herrero, Baykal-Gürsoy \& Jaśkiewicz [31]), setting the price under a mean variance framework.

Liyanage \& Shanthikumar [32] propose an approach called operational statistics, which integrates the tasks of parameter estimation and optimization (maximizing expected profit for the single period news-vendor model). When no assumptions are made about the form of the demand distribution, Chu, Shanthikumar \& Shen [33] derive operational statistics that maximize the performance uniformly for all values of the unknown demand parameters using Bayesian analysis.

To set the stage for introducing our innovation, we first delve into the basics of inventory theory in Lemma 1 and Lemma 2. The solution, based on our innovation, is not to find an optimal quantity to order, but to find an optimal distribution, from which a sample is drawn to give the order quantity, with the objective of maximizing the expected profit. The key findings are outlined in Theorem 1 and Proposition 1, which are novel, to the best of our knowledge.

\subsection{Notation and Terminology}

Let $Q$ represent the amount ordered by the retailer before the selling season starts. Let, the unit cost to manufacture the product be $c$, the wholesale price at which manufacturer produces and sells to the retailer be $w$, the salvage value of any unsold product is $s$ per unit, and the stockout cost of unsatisfied demand is $r$ per unit. The final price at which retailer sells is $p$. Except in the benchmark model, to avoid unrealistic and trivial cases, we assume that $0<s<w<p$, $p>c$ and $0<r$. Retailer faces stochastic demand, $D$, with Cumulative distribution $F$ and density $f$. When $Q$ is stochastic, it has cumulative distribution $G$ and density $g$. We assume that $F, G$ are continuous and strictly increasing functions and $f, g$ are non-negative functions. $Q$ and $D$ have joint distribution function $F_{Q, D}(q, d) \Leftrightarrow F_{X, Y}(x, y)$ and joint density function 
$f_{Q, D}(q, d) \Leftrightarrow f_{X, Y}(x, y)$. Notice that we write $X$ for $Q$ and $Y$ for $D$ to prevent confusions later when we work with calculus notations. We further assume $Q$ and $D$ are independent giving, $f_{X, Y}(x, y)=g(x) f(y)$ and $F_{X, Y}(x, y)=G(x) F(y)$. We will relax this assumption of independence and assume that the covariance between the processes is given by, $\sigma_{X Y}$ in subsequent papers (Kashyap [34]).

- $D \sim F[0, u], D$ is distributed according to the cumulative distribution $F$ over the interval $[0, u]$.

- $F$, is increasing and has full support, which is the non-negative real line $[0, \infty]$.

- $f=F^{\prime}$, is the continuous density function of $F$.

- $D \sim U[a, b]$, if we consider the uniform distribution.

- $D \sim L N[0, u]$, if we consider the log normal distribution.

- $Q \sim G[0, v], Q$ is distributed according to the cumulative distribution $G$ over the interval $[0, v]$.

- $G$, is increasing and has full support, which is the non-negative real line $[0, \infty]$.

- $g=G^{\prime}$, is the continuous density function of $G$.

- $Q \sim U[u, v]$, if we consider the uniform distribution.

- $Q \sim L N[0, v]$, if we consider the log normal distribution.

- $Q, D \sim F_{X, Y}(x, y), Q$ and $D$ have joint distribution function $F_{X, Y}(x, y)=F(x) G(y)$ and joint density function $f_{X, Y}(x, y)=g(x) f(y)$ because of independence. When we relax the assumption of independence, $\operatorname{cov}(Q, D)=\operatorname{cov}(X, Y)=\sigma_{X Y}$.

- $\forall a, b \in \Re_{+}, a^{+}=\max \{a, 0\}, a^{-}=\max \{-a, 0\}$, and $a \wedge b=\min \{a, b\}$.

- $\pi_{R}$, the expected profits of the retailer when only Demand, $D$, is stochastic and $Q$ the quantity ordered is the control variable.

- $Q^{*}$, the optimal quantity to order when only Demand, $D$, is stochastic, under the true distribution, $F(y)$.

- $\pi_{R}^{*}$, the optimal expected profits of the retailer when only Demand, $D$, is stochastic and $Q$ the quantity ordered is the control variable, under the true distribution, $F(y)$.

- $\pi_{R S}$, the expected profits of the retailer when Demand, $D$, is stochastic and $Q$ the quantity ordered is also stochastic.

- $\tilde{F}(y), \tilde{f}(y)$, the estimated demand distribution and density, which will be different from the actual distribution, $F(y) \neq \tilde{F}(y) \Rightarrow f(y) \neq \tilde{f}(y)$, due to measurement errors and other issues.

- $\hat{F}(y), \hat{f}(y)$, the compound demand distribution and density, with the assumption that the parameters of the estimated distribution, $\tilde{F}(y)$, have distributions of their own, due to measurement errors and other issues.

- $\hat{F}_{X, Y}(x, y)=G(x) \hat{F}(y), \hat{f}_{X, Y}(x, y)=g(x) \hat{f}(y)$, are the joint distribution function and joint density function of the compound demand process, $\hat{F}(y), \hat{f}(y)$, and the stochastic quantity ordered.

- $\hat{Q}^{*}$, the actual quantity that will be ordered, with the optimization performed 
using the estimated distribution, $\tilde{F}(y)$.

- $\hat{\pi}_{R}^{*}$, the actual expected profits under the compound distribution, $\hat{F}(y)$ when the quantity ordered is $\hat{Q}^{*}$.

\subsection{Benchmark Model}

Before we look at stochastic variations in the quantity to be ordered, we collect results when the retailer optimizes his profit function to obtain the optimal amount, since it offers a comparison point for the later scenarios. We begin with the simplest possible scenario by setting $s=r=0$. This assumption implies there is no stockout costs (no loss of goodwill or direct noticeable cost) if a demand is not fulfilled by the retailer to both the manufacturer and retailer and there is no salvage value. We assume that this is a full information scenario, that is a game in which both parties know about the constraints and incentives that the other party faces and is looking to optimize in what environment. The retailer maximizes his profit based on the following criteria,

$$
\begin{aligned}
& \pi_{R}= \begin{cases}\{Q p-Q w\}, & \text { if }(D>Q) \\
\{D p-Q w\}, & \text { else if }(D<Q)\end{cases} \\
& E\left(\pi_{R}\right)=E_{D}[\min (Q, D) p-Q w] \\
& \pi_{R}^{*}=\max _{Q} E_{D}[\min (Q, D) p-Q w]
\end{aligned}
$$

Lemma 1. The mean and variance of the profits are given by,

$$
\begin{gathered}
E\left(\pi_{R}\right)=(p-w) Q-p \int_{0}^{Q} F(t) \mathrm{d} t \\
\operatorname{Var}\left(\pi_{R}\right)=p^{2}\left[\int_{0}^{Q} 2(Q-t) F(t) \mathrm{d} t-\left\{\int_{0}^{Q} F(t) \mathrm{d} t\right\}^{2}\right]
\end{gathered}
$$

Proof. See Appendix A.

Lemma 2. The optimal quantity to order, the optimal expected profits of the retailer and the variance when the optimal quantity is ordered are given by,

$$
\begin{gathered}
Q^{*}=F^{-1}\left(1-\frac{w}{p}\right) \\
\pi_{R}^{*}=Q^{*}(p-w)-p \int_{0}^{Q^{*}} F(t) \mathrm{d} t=p \int_{0}^{Q^{*}} t f(t) \mathrm{d} t \\
\pi_{R}^{*}=p \int_{0}^{Q^{*}} P(D>t) \mathrm{d} t-Q^{*} w \\
\operatorname{Var}\left(\pi_{R}^{*}\right)=p^{2}\left[\int_{0}^{Q^{*}} 2\left(Q^{*}-t\right) F(t) \mathrm{d} t-\left\{\int_{0}^{Q^{*}} F(t) \mathrm{d} t\right\}^{2}\right] \\
\operatorname{Var}\left(\pi_{R}^{*}\right)=p^{2}\left[\left(Q^{*}\right)^{2}\left\{1-\left(\frac{w}{p}\right)^{2}\right\}-\left(\int_{0}^{Q^{*}} t f(t) \mathrm{d} t\right)^{2}\right. \\
\left.-2 Q^{*} \frac{w}{p} \int_{0}^{Q^{*}} t f(t) \mathrm{d} t-2 \int_{0}^{Q^{*}} t F(t) \mathrm{d} t\right]
\end{gathered}
$$

Proof. See Appendix B. 


\subsection{Stochastic Quantity Ordered}

Any distribution, $\tilde{F}(y)$, we estimate for the demand will be different from the true distribution, $F(y) \neq \tilde{F}(y) \Rightarrow f(y) \neq \tilde{f}(y)$, due to measurement errors, change in preferences of agents and other matters that make predictions go awry. This means that the parameters of the estimated distribution can be further assumed to have distributions of their own. A reasonable assumption can be that the parameters of the estimated distribution are normal (or any other suitable one), making the demand distribution a compound distribution, $\hat{F}(y)$ with density $\hat{f}(y)$. Hence the actual quantity we order, $\hat{Q}^{*}$, could be different from the true optimal quantity, $\hat{Q}^{*} \neq Q^{*}$, and and hence the profits that actually accrue, $\hat{\pi}_{R}^{*}$, could be lower than the true optimal profits, $\hat{\pi}_{R}^{*} \leq \pi_{R}^{*}$.

It is important to keep in mind that, the actual quantity ordered, $\hat{Q}^{*}$, is the result of an optimization performed using the estimated distribution, $\tilde{F}(y)$; but the profits that will accrue, $\hat{\pi}_{R}^{*}$, when we order this quantity, $\hat{Q}^{*}$, is the result obtained when operating in an environment where the compound distribution, $\hat{F}(y)$, holds influence. To counter this measurement error, we introduce randomness in the number of items ordered. This means, $Q$ representing the amount ordered by the retailer, before the selling season starts, will be stochastic, with cumulative distribution $G$ and density $g . Q$ and $D$ have joint distribution function $\hat{F}_{X, Y}(x, y)=G(x) \hat{F}(y)$ and joint density function $\hat{f}_{X, Y}(x, y)=g(x) \hat{f}(y)$ because of independence.

Theorem 1. When the quantity ordered is stochastic: the expected profits, $E\left[\pi_{R S}\right]$, will be greater than, or equal to, the expected profits of the benchmark model $\hat{\pi}_{R}^{*}$, such that the condition, $E\left[\pi_{R S}\right] \geq \hat{\pi}_{R}^{*}$ is satisfied, ensuring that this approach will yield better outcomes, as long as the following restriction holds on the parameters.

$$
\begin{gathered}
E[Q]\left[1-\frac{w}{p}\right]+E[D]-E[\max (Q, D)] \geq \int_{0}^{\hat{Q}^{*}} t \hat{f}(t) \mathrm{d} t \\
\left\{\text { Here, } \hat{Q}^{*}=\tilde{F}^{-1}\left(1-\frac{w}{p}\right)\right\}
\end{gathered}
$$

The expected profits, $E\left[\pi_{R S}\right]$, are with resect to the joint distribution,

$$
\hat{F}_{X, Y}(x, y)=G(x) \hat{F}(y)
$$

where demand is governed by the compound distribution, $\hat{F}(y)$. The expected profits of the benchmark model, $\hat{\pi}_{R}^{*}$, are with respect to the compound distribution, $\hat{F}(y)$, when the actual quantity ordered, $\hat{Q}^{*}$, is obtained by optimizing under the estimated distribution, $\tilde{F}(y)$.

Proof. See Appendix C.

Instead of optimizing the quantity ordered with respect to the compound distribution of demand with measurement errors, randomizing is better due to the errors in measurement errors and so on, ad infinitum (Taleb [35]). All of these errors are extremely hard to forecast, considering that a big unknown is how many recursive levels of errors we might encounter, or, what is a satisfactory 
level to stop.

A central question is: how will buyers respond to different inventory management techniques. DeGraba [36] investigates tactics employed by sellers to induce excess demand, (leading to higher prices), by creating artificial shortages. The message from the usage of such methods is that, we can randomize across a subset of states for the quantity ordered, or, pick randomly from a few of the most likely possibilities, (or the desired set, that need not be the most likely), for the quantity ordered. This can also be understood as optimizing to get an interval of acceptable performance and randomizing over that interval; given our questionable ability to get the best outcome, we go with a mix of the better outcomes. Hence, the prescription, "Don't Simply Optimize, Also Randomize; perhaps best described by the term-Randoptimization". Since we control the choice of the distribution for $Q$, we note a nice property in the following proposition.

Proposition 1. The parameters of the distribution for the stochastic quantity ordered can be got by solving the below expression, if its expected value is set such that $E[Q]=\hat{Q}^{*}$.

$$
\int_{0}^{\infty} x g(x) \hat{F}(x) \mathrm{d} x+\int_{0}^{\infty} y \hat{f}(y) G(y) \mathrm{d} y \leq \hat{Q}^{*} \hat{F}\left(\hat{Q}^{*}\right)+\int_{\hat{Q}^{t}}^{\infty} \hat{f}(t) \mathrm{d} t
$$

\section{Proof. See Appendix D.}

The result in proposition 1 can be viewed as an extra set of constraints on the parameters of the distribution, from which we have to choose the quantity to order. This simplification has an intuitive appeal that the expected value of the stochastic quantity ordered is equal to the optimal estimation of the quantity to order, in the presence of measurement errors. Depending on the distribution for the demand and the distribution from which we sample the quantity to order, this criteria can turn out to be highly restrictive, in which case the more relaxed results from theorem 1 need to be used instead.

\section{Discussion of Other Applications}

We provide a brief synopsis of how this concept can be useful in other areas, with a complete development of each sub-topic planned in subsequent papers.

\subsection{School Admissions}

University rankings and the rush to get in to the best schools, as determined by these rankings, is well acknowledged and studied. The prestige associated with having attended a top school lingers on for a lifetime and beyond. The admission process and how valid or effective it is in determining determining academic success or corporate productivity are debatable at best: (Fishman and Pasanella [37]; Deckro \& Woundenberg [38]; Willingham [39]; Sorensen [40]; Sandow, Jones, Peek, Courts \& Watson [41]).

While aspirations for higher rankings and the quest for excellence are worthy pursuits, what rankings have created are false targets and misleading perceptions. The goal should not simply be to get into the best possible school, but to get into 
a good school and get the best possible education. No doubt, admission to an elite university can help obtain a great education, but the difference in the quality of teaching at most good universities is not that significant. Without going into too many details, we mention that people go to great lengths simply to secure admission into the top colleges. The current set up of rankings and the best ranked institutions attracting the best minds and resources, become self fulfilling prophecies that segment society, which is one of the primary outcomes that education seeks to eradicate.

Conjecture 3. When making offers of admission to schools, it can be made publicly known that the selection will be done at random from a pool of candidates who meet a certain number of basic qualifying criteria. Different universities could even collaborate to select students from the overall pool in this way, after considering applicable constraints and preferences. This will ensure that the rankings will change as the world changes and the focus will not be on getting into the best university, but to get into a good university and getting the best possible education.

For simplicity, the discussion above does not distinguish between admission to universities for undergraduate education and for admission into elementary and high schools, since the same solution can apply to all these cases with minor adjustments. Postgraduate education can also use these methods, but a greater level of restrictions needs to be placed, to match the preferences of candidates regarding the desired specialized field of study and the strengths of the higherlevel educational institution. Of course, certain exceptions can always be made, wherein, really deserving candidates and truly exceptional students can be granted admission, without subjecting them to the above procedure.

\subsection{Job Candidate Selection}

Admissions to schools is still a much more open and fair process when compared to the job market, where cronyism, connections and catchy phrases written on resumes, which manage to get the attention of hiring managers, seem to be the key. A similar random process could select the successful candidate from the pool of applicants meeting certain benchmark criteria. Of course, human resource personnel, recruiters and hiring managers who pride themselves on finding the perfect fit for their organization would voice serious concerns. We are not looking to malign or undermine that effort. But would it really make such a big difference how perfect the candidate as long as he is not a drug addict or a violent criminal and he can learn the skills required to perform the job once hired? Human resources can then focus on training programs and other means of actually enhancing productivity.

\subsection{Journal Submissions}

A topic that would perhaps determine the fate of this paper and others we plan to write developing these topics is about the current selection process at the top journals. But when papers such as these are being written, [the titles of these 
papers are quite sufficient to understand the nature of the issues they address: (Zivney \& Bertin [42]), "Publish or perish: What the competition is really doing"; (Seglen [43]), "Why the impact factor of journals should not be used for evaluating research"; (Choi [44]), "How to publish in top journals"; (Lawrence [45]), "The politics of publication"; (De Rond \& Miller [46]), "Publish or Perish: Bane or Boon of Academic Life?"], it is a warning sign that maximum efforts are not being spent on creating the best work, but a lot of work is spent on getting published at the top outlets.

With no offense to any of the editors and reviewers who spend countless hours finding the most suitable papers for their journals. Would it not be easier and better to randomly select good papers from the overall pool of submissions and coach the authors so that the papers can develop to become excellent papers? Different journals could even collaborate to select papers from the submission pool in this way. This will perhaps also ensure that papers will not just be judged merely by where they are published but a deeper evaluation will be done by future authors to determine what previous work is helpful for them. It can be argued that such a thorough assessment will lead to better fulfillment of one of the fundamental goals of research and the pursuit of knowledge, which is to promote better decision making. No doubt, the problem here is not that severe since the best works do bubble up to the surface over time and researchers are careful in selecting the works they deem beneficial for further studying or enhancing. We merely include this example as another instance where introducing uncertainty in the decision process can yield better outcomes over time.

\subsection{Stock Picking}

No discussion on uncertainty in the social sciences is complete without talking about the financial markets. The amount of effort devoted to security selection and chasing higher returns is colossal. A simple mechanism could pick a pool of securities meeting certain criteria and randomly allocate wealth to a smaller percentage of the overall pool. This would acknowledge the randomness (Taleb [47]) inherent in the outcomes of star portfolio managers and re-channel efforts from financial management to other more worthy endeavors.

\subsection{Capitol Hill Baby Sitting Crisis and Monetary Policy Management}

Lastly, we consider, perhaps, one of the most elegant, simple and beautiful examples of uncertainty in social systems. The (Sweeney \& Sweeney [48]) anecdote about the Capitol Hill baby-sitting crisis exposits the mechanics of inflation, setting interest rates and monetary policies required to police the optimum amount of money. The creation of a monetary crisis in a small, simple environment of good-hearted people, expounds that even with near ideal conditions, things can become messy; then in a large labyrinthine atmosphere, disaster could be brewing without getting noticed and can strike without much premonition. 
The primary emphasis should be on the need to keep complexity at bay and establishing an ambience, where repeated games can be played with public transparency, so that guileful practices can be curtailed. A solution based on the central theme of our paper, could be to issue lotteries and award scrips (fiat money that could be exchanged in lieu of baby sitting hours) to the residents when there is a recession (too many baby sitters and not enough baby sitting jobs or money to be made) and expire a certain amount of scrips either periodically or even better on randomly selected dates when there are inflationary pressures (too much money or scrip chasing too few good baby sitters). The simplicity of our solution is a stark contrast to the elegant yet complex approach, filled with assumptions, outlined in (Hens, Schenk-Hoppé \& Vogt [49]). A detailed comparison and related treatments are postponed to another time.

\section{Limitations and Scope for Further Research}

In the interest of brevity, we have only provided initial results for the inventory management case and briefly discussed other examples.

- The inventory management problem can be extended to consider example distributions and estimation of parameters that can satisfy the inequalities derived (Brunk [50]; Parzen [51]; Nickalls [52]; Jaakkola \& Jordan [53]; Gibbs \& Su [54]; Weisstein [55]). The hunt for distributions for the quantity to order can be quite laborious and could involve both discrete and continuous varieties. When no solutions exist or when solutions are not easily obtained, a limited number of nearly optimal states need be identified and their profit potential ascertained, keeping in mind the extent of maximum losses that could be incurred.

- The main result in Theorem 1 is simplistic in nature, but is mostly meant to illustrate that our approach, where the quantity ordered is stochastic, holds promise, and further research in this direction can be rewarding.

- Also for simplicity, we have assumed independence between the demand process and the stochastic quantity ordered. The number of feasible solutions in terms of the distribution parameters for the stochastic quantity might be limited or not be easily obtained in this case. We can assume covariance between these two random variables to obtain more valid solutions. We might expect that when the two variables are positively correlated, the output, in terms of profits, might be more beneficial. These results, need to be established theoretically and also empirically verified, to provide guidance on the choice of distributions for the quantity to be ordered and the extent of correlation with the demand process.

- A deeper analysis could yield a useful set of comparisons, that establish bounds on the difference in profits under the true demand distribution, the estimated demand distribution (a good example of which is the empirical distribution function) and the scenario when the quantity ordered is stochastic. Such bounds under both finite sample situations and asymptotically, could shed light on the criteria when the stochastic quantity would be 
a beneficial choice.

- A realistic assumption would be that extreme demand situations or outliers would be underrepresented in a historical time series of observed demand. It would be interesting to understand the behavior of the profits when the variance of the demand distribution varies and when outliers occur. The choice of the distribution for the stochastic quantity to order should aim to push the system into regions where, despite any errors in estimation, profits are not significantly affected and if possible even benefit from the fluctuations of the demand process.

- Different utility functions or mean variance based optimization techniques can also be introduced into this framework.

- We are working on creating subsequent papers to significantly enhance the results for inventory management, to develop theoretical platforms and test empirically the other case studies mentioned here.

The dynamic nature of any social-science system, where changes can be observed and decisions can be made by participants to influence the system, means that the limited predictive ability of any awareness will necessitate periodic reviews and the prescription of corrective programs. It would be interesting to see how participants modify their actions once randomness is introduced in the decision making process. Where there is uncertainty, there will be unintended consequences, which might be welcome or hazardous (Kashyap [56]). Of interest would be to see whether such an approach will reduce the sense of entitlement prevalent in society or whether it would lead to greater complacency.

\section{Conclusions}

We have developed a framework using the inventory management example, to illustrate how we can overcome uncertainty with uncertainty. Using randomness in our choices and in what we control, and hence in the decision making process, we could potentially offset the uncertainty inherent in the environment and yield better outcomes. Such an approach, while seemingly absurd, is the ideal medicine for the even greater absurdity in the decision making process, which has become prevalent in today's society. It takes care of the issues that crop up due to the limitations in our understanding of complex systems and the widely acknowledged problem that most of our measurements are highly prone to errors.

The central innovation can be understood as optimizing to get an interval of adequate performance and randomizing over that interval. The goal is not just to optimize, but to identify a region of optimal performance and set the parameters of a probability distribution over that region and sample from it to provide a satisfactory level of performance. Agents looking to optimize will randomize over an optimal region, hence this approach is called "Randoptimization". Due to measurement errors and other uncertainty, we can never be certain of any optimization we perform, rather it is better to randomize over acceptable states.

We have discussed areas where such an approach might be suited, with the 
common prescription, "Don't Simply Optimize, Also Randomize; perhaps best described by the term-Randoptimization".

1) News-Vendor Inventory Management Problem

2) School Admissions

3) Journal Submissions

4) Job Candidate Selection

5) Stock Picking

6) Monetary Policy

\section{References}

[1] Keeney, R.L. (1982) Decision Analysis: An Overview. Operations Research, 30, 803838. https://doi.org/10.1287/opre.30.5.803

[2] Pratt, J.W., Raiffa, H. and Schlaifer, R. (1964) The Foundations of Decision under Uncertainty: An Elementary Exposition. Journal of the American Statistical Association, 59, 353-375. https://doi.org/10.1080/01621459.1964.10482164

[3] Raiffa, H. (2002) Decision Analysis: A Personal Account of How It Got Started and Evolved. Operations Research, 50, 179-185. https://doi.org/10.1287/opre.50.1.179.17797

[4] Ramsey, F.P. (1926) Truth and Probability. Histoy of Economic Thought Chapters, 57, 211-238.

[5] Savage, L.J. (1954) The Foundations of Statistics. Dialectica, 8, 95-111. https://doi.org/10.1111/j.1746-8361.1954.tb01124.x

[6] Kashyap, R. (2017) Microstructure under the Microscope: Tools to Survive and Thrive in The Age of (Too Much) Information. The Journal of Trading, 12, 5-27. https://doi.org/10.3905/jot.2017.12.2.005

[7] Kashyap, R. (2014) Dynamic Multi-Factor Bid-Offer Adjustment Model. The Journal of Trading, 9, 42-55. https://doi.org/10.3905/jot.2014.9.3.042

[8] Lawson, T. (1985) Uncertainty and Economic Analysis. The Economic Journal, 95, 909-927. https://doi.org/10.2307/2233256

[9] Keynes, J.M. (1937) The General Theory of Employment. The Quarterly Journal of Economics, 51, 209-223. https://doi.org/10.2307/1882087

[10] Keynes, J.M. (1971) The Collected Writings of John Maynard Keynes: In 2 Volumes. A Treatise on Money. The Applied Theory of Money. Macmillan for the Royal Economic Society.

[11] Keynes, J.M. (1973) A Treatise on Probability, the Collected Writings of John Maynard Keynes, Vol. VIII.

[12] Knight, F.H. (1921) Risk, Uncertainty and Profit. Houghton Mifflin Company, Boston, 682-690.

[13] Hoogduin, L. (1987) On the Difference between the Keynesian, Knightian and the "Classical" Analysis of Uncertainty and the Development of a More General Monetary Theory. De Economist, 135, 52-65. https://doi.org/10.1007/BF01856703

[14] McManus, H. and Hastings, D. (2005) 3.4. 1 A Framework for Understanding Uncertainty and its Mitigation and Exploitation in Complex Systems. INCOSE International Symposium, 15, 484-503.

[15] Kashyap, R. (2015) A Tale of Two Consequences. The Journal of Trading, 10, 51 95. https://doi.org/10.3905/jot.2015.10.4.051

[16] Kashyap, R. (2016) Hong Kong-Shanghai Connect/Hong Kong-Beijing Disconnect, 
Scaling the Great Wall of Chinese Securities Trading Costs. The Journal of Trading, 11, 81-134. https://doi.org/10.3905/jot.2016.11.3.081

[17] Simon, H.A. (1962) The Architecture of Complexity. Proceedings of the American Philosophical Society, 106, 467-482.

[18] Kashyap, R. (2015) Financial Services, Economic Growth and Well-Being. Indian Journal of Finance, 9, 9-22. https://doi.org/10.17010/ijf/2015/v9i1/71531

[19] Kashyap, R. (2017b) Solving Society's Big Ills, A Small Step. Asian Social Science, 13, 175-191. https://doi.org/10.5539/ass.v13n4p175

[20] Kashyap, R. (2016) Combining Dimension Reduction, Distance Measures and Covariance. Social Science Research Network (SSRN). Working Paper.

[21] Kleywegt, A.J. and Shapiro, A. (2001) Stochastic Optimization. Handbook of Industrial Engineering. Springer-Verlag, Berlin, 2625-2649. https://doi.org/10.1002/9780470172339.ch102

[22] Arrow, K.J., Harris, T. and Marschak, J. (1951) Optimal Inventory Policy. Econometrica: Journal of the Econometric Society, 19, 250-272. https://doi.org/10.2307/1906813

[23] Chen, F. and Federgruen, A. (2000) Mean-Variance Analysis of Basic Inventory Models. Columbia University, New York.

[24] Cachon, G.P. and Lariviere, M.A. (2005) Supply Chain Coordination with RevenueSharing Contracts: Strengths and Limitations. Management Science, 51, 30-44. https://doi.org/10.1287/mnsc.1040.0215

[25] Khouja, M. (1999) The Single-Period (News-Vendor) Problem: Literature Review and Suggestions for Future Research. Omega, 27, 537-553. https://doi.org/10.1016/S0305-0483(99)00017-1

[26] Qin, Y., Wang, R., Vakharia, A.J., Chen, Y. and Seref, M.M. (2011) The NewsVendor Problem: Review and Directions for Future Research. European Journal of Operational Research, 213, 361-374. https://doi.org/10.1016/j.ejor.2010.11.024

[27] Gallego, G. and Moon, I. (1993) The Distribution Free Newsboy Problem: Review and Extensions. Journal of Operational Research Society, 44, 825-834.

https://doi.org/10.1057/jors.1993.141

[28] Gallego, G. and Van Ryzin, G. (1994) Optimal Dynamic Pricing of Inventories with Stochastic Demand over Finite Horizons. Management Science, 40, 999-1020. https://doi.org/10.1287/mnsc.40.8.999

[29] Wu, J., Li, J., Wang, S. and Cheng, T.C.E. (2009) Mean-Variance Analysis of the News-Vendor Model with Stockout Cost. Omega, 37, 724-730. https://doi.org/10.1016/j.omega.2008.02.005

[30] Wilson, J.G. and Sorochuk, C. (2013) The News-Vendor Problem with Pricing and Secondary Revenues. Journal of Applied Business and Economics, 14, 11-23.

[31] Rubio-Herrero, J., Baykal-Gürsoy, M. and Jaśkiewicz, A. (2015) A Price-Setting News-Vendor Problem under Mean-Variance Criteria. European Journal of Operational Research, 247, 575-587. https://doi.org/10.1016/j.ejor.2015.06.006

[32] Liyanage, L.H. and Shanthikumar, J.G. (2005) A Practical Inventory Control Policy Using Operational Statistics. Operations Research Letters, 33, 341-348. https://doi.org/10.1016/j.orl.2004.08.003

[33] Chu, L.Y., Shanthikumar, J.G. and Shen, Z.J.M. (2008) Solving Operational Statistics via a Bayesian Analysis. Operations Research Letters, 36, 110-116. https://doi.org/10.1016/j.orl.2007.04.010

[34] Kashyap, R. (2015) Fighting Uncertainty with Uncertainty. Social Science Research Network (SSRN). Working Paper. 
[35] Taleb, N.N. (2012) The Future Has Thicker Tails than the Past: Model Error as Branching Counterfactuals. arXiv preprint arXiv:1209.2298.

[36] DeGraba, P. (1995) Buying Frenzies and Seller-Induced Excess Demand. The RAND Journal of Economics, 26, 331-342. https://doi.org/10.2307/2555920

[37] Fishman, J.A. and Pasanella, A.K. (1960) College Admission-Selection Studies. Review of Educational Research, 30, 298-310. https://doi.org/10.2307/1168852

[38] Deckro, R.F. and Woundenberg, H.W. (1977) MBA Admission Criteria and Academic Success. Decision Sciences, 8, 765-769. https://doi.org/10.1111/j.1540-5915.1977.tb01120.x

[39] Willingham, W.W. (1985) Success in College: The Role of Personal Qualities and Academic Ability. College Board Publications, New York.

[40] Sorensen, C.W. (1994) Success and Education in South Korea. Comparative Education Review, 38, 10-35. https://doi.org/10.1086/447223

[41] Sandow, P.L., Jones, A.C., Peek, C.W., Courts, F.J. and Watson, R.E. (2002) Correlation of Admission Criteria with Dental School Performance and Attrition. Journal of Dental Education, 66, 385-392.

[42] Zivney, T.L. and Bertin, W.J. (1992) Publish or Perish: What the Competition Is Really Doing. The Journal of Finance, 47, 295-329.

https://doi.org/10.1111/j.1540-6261.1992.tb03987.x

[43] Seglen, P.O. (1997) Why the Impact Factor of Journals Should Not Be Used for Evaluating Research. The BMJ, 314, 497. https://doi.org/10.1136/bmj.314.7079.497

[44] Choi, K. (2005) How to Publish in Top Journals Part I. Pan-Pacific Management Review, 8, 91-106.

[45] Lawrence, P.A. (2003) The Politics of Publication. Nature, 422, 259-261. https://doi.org/10.1038/422259a

[46] De Rond, M. and Miller, A.N. (2005) Publish or Perish: Bane or Boon of Academic Life? Journal of Management Inquiry, 14, 321-329. https://doi.org/10.1177/1056492605276850

[47] Taleb, N. (2005) Fooled by Randomness: The Hidden Role of Chance in Life and in the Markets. Random House, New York.

[48] Sweeney, J. and Sweeney, R.J. (1977) Monetary Theory and the Great Capitol Hill Baby Sitting Co-Op Crisis: Comment. Journal of Money, Credit and Banking, 9, 8689. https://doi.org/10.2307/1992001

[49] Hens, T., Schenk-Hopp, K.R. and Vogt, B. (2007) The Great Capitol Hill Baby Sitting Co-Op: Anecdote or Evidence for the Optimum Quantity of Money? Journal of Money, Credit and Banking, 39, 1305-1333. https://doi.org/10.1111/j.1538-4616.2007.00068.x

[50] Brunk, H.D. (1958) On the Estimation of Parameters Restricted by Inequalities. The Annals of Mathematical Statistics, 29, 437-454. https://doi.org/10.1214/aoms/1177706621

[51] Parzen, E. (1962) On Estimation of a Probability Density Function and Mode. The Annals of Mathematical Statistics, 33, 1065-1076. https://doi.org/10.1214/aoms/1177704472

[52] Nickalls, R.W.D. (1993) A New Approach to Solving the Cubic: Cardan's Solution Revealed. The Mathematical Gazette, 77, 354-359. https://doi.org/10.2307/3619777

[53] Jaakkola, T.S. and Jordan, M.I. (2000) Bayesian Parameter Estimation via Variational Methods. Statistics and Computing, 10, 25-37. https://doi.org/10.1023/A:1008932416310

[54] Gibbs, A.L. and Su, F.E. (2002) On Choosing and Bounding Probability Metrics. 
International Statistical Review, 70, 419-435.

https://doi.org/10.1111/j.1751-5823.2002.tb00178.x

[55] Weisstein, E.W. (2002) Cubic Formula. Omega, 86, 87.

[56] Kashyap, R. (2016) Notes on Uncertainty, Unintended Consequences and Everything Else. Working Paper. Social Science Electronic Publishing.

[57] Kungwani, P. (2014) Risk Management-An Analytical Study. IOSR Journal of Business and Management, 16, 83-89. https://doi.org/10.9790/487X-16338389 


\section{Appendix A: Proof of Lemma 1}

Proof. The proof is standard and well known; but we provide it because of its central importance to this paper and for completeness. Consider the profit function of the benchmark model,

$$
\begin{aligned}
& \pi_{R}=\min (Q, D) p-Q w \\
& \pi_{R}=\left\{Q-(Q-D)^{+}\right\} p-Q w \\
& \left\{\because \min (Q, D)=\left\{Q-(Q-D)^{+}\right\}\right. \text {; } \\
& \text { If }(Q<D) ;\left\{Q-(Q-D)^{+}\right\}=Q-0=\min (Q, D) \\
& \text { If } \left.(Q \geq D) ;\left\{Q-(Q-D)^{+}\right\}=Q-(Q-D)=D=\min (Q, D)\right\} \\
& \pi_{R}=(p-w) Q-p(Q-D)^{+} \\
& E\left(\pi_{R}\right)=E_{D}\left[(p-w) Q-p(Q-D)^{+}\right] \\
& E\left(\pi_{R}\right)=(p-w) Q-p \int_{0}^{Q} F(t) \mathrm{d} t \\
& \left\{\because E_{D}\left[p(Q-D)^{+}\right]=p \int_{0}^{Q}(Q-t) f(t) \mathrm{d} t=p Q \int_{0}^{Q} f(t) \mathrm{d} t-p \int_{0}^{Q} t f(t) \mathrm{d} t ;\right. \\
& \text { Integrating by Parts, using, } \int u \mathrm{~d} v=u v-\int v \mathrm{~d} u \text {, } \\
& \left.p \int_{0}^{Q} t f(t) \mathrm{d} t=p Q \int_{0}^{Q} f(t) \mathrm{d} t-p \int_{0}^{Q} F(t) \mathrm{d} t ; p Q \int_{0}^{Q} f(t) \mathrm{d} t=p Q F(Q)\right\} \\
& \operatorname{Var}\left(\pi_{R}\right)=E\left[\pi_{R}-E\left(\pi_{R}\right)\right]^{2}=E\left[\left(\pi_{R}\right)^{2}\right]-\left[E\left(\pi_{R}\right)\right]^{2} \\
& \operatorname{Var}\left(\pi_{R}\right)=E_{D}\left[(p-w)^{2} Q^{2}+p^{2}\left\{(Q-D)^{+}\right\}^{2}-2\left\{(p-w) p Q(Q-D)^{+}\right\}\right] \\
& -\left[(p-w)^{2} Q^{2}+p^{2}\left\{\int_{0}^{Q} F(t) \mathrm{d} t\right\}^{2}-2\left\{(p-w) p Q \int_{0}^{Q} F(t) \mathrm{d} t\right\}\right] \\
& \operatorname{Var}\left(\pi_{R}\right)=E_{D}\left[p^{2}\left\{(Q-D)^{+}\right\}^{2}\right]-p^{2}\left\{\int_{0}^{Q} F(t) \mathrm{d} t\right\}^{2} \\
& \operatorname{Var}\left(\pi_{R}\right)=p^{2}\left[\int_{0}^{Q} 2(Q-t) F(t) \mathrm{d} t-\left\{\int_{0}^{Q} F(t) \mathrm{d} t\right\}^{2}\right] \\
& \left\{\because E_{D}\left[\left\{(Q-D)^{+}\right\}^{2}\right]=\int_{0}^{Q}(Q-t)^{2} f(t) \mathrm{d} t\right. \text {, Integrate this by Parts } \\
& \left.=\left|(Q-t)^{2} F(t)\right|_{0}^{Q}-\int_{0}^{Q} 2(Q-t)(-1) F(t) \mathrm{d} t\right\}
\end{aligned}
$$

\section{Appendix B: Proof of Lemma 2}

Proof. The proof is standard and well known; but we provide it because of its central importance to this paper and for completeness. Consider maximizing the profit function of the benchmark model,

$$
\begin{aligned}
\pi_{R}^{*} & =\max _{Q} E_{D}[\min (Q, D) p-Q w] \\
& =\max \left[\int_{0}^{Q} p t f(t) \mathrm{d} t+\int_{Q}^{\infty} Q p f(t) \mathrm{d} t-Q w\right]
\end{aligned}
$$




$$
\left\{\text { we write this instead of } \max \left[\int_{0}^{Q} p d f(d) \mathrm{d} d+\int_{Q}^{\infty} Q p f(d) \mathrm{d} d-Q w\right]\right\}
$$

First Order Conditions using Leibniz Integral Rule gives,

$$
\begin{gathered}
\frac{\partial\left[\int_{0}^{Q} p t f(t) \mathrm{d} t+\int_{Q}^{\infty} Q p f(t) \mathrm{d} t-Q w\right]}{\partial Q}=0 \\
p Q f(Q)+\int_{Q}^{\infty} p f(t) \mathrm{d} t-Q p f(Q)-w=0 \\
\Rightarrow\{F(\infty)-F(Q)\}=\frac{w}{p} \\
F(Q)=1-\frac{w}{p}
\end{gathered}
$$

This gives the optimal quantity to order for the retailer as,

$$
Q^{*}=F^{-1}\left(1-\frac{w}{p}\right)
$$

The optimal expected profits of the retailer would then be

$$
\begin{gathered}
\pi_{R}^{*}=E_{D}\left[\min \left(Q^{*}, D\right) p-Q^{*} w\right] \\
\pi_{R}^{*}=p \int_{0}^{Q^{*}} t f(t) \mathrm{d} t+\int_{Q^{*}}^{\infty} Q^{*} p f(t) \mathrm{d} t-Q^{*} w \\
\pi_{R}^{*}=p \int_{0}^{Q^{*}} t f(t) \mathrm{d} t+Q^{*} p\left\{1-\int_{0}^{Q^{*}} f(t) \mathrm{d} t\right\}-Q^{*} w \\
\pi_{R}^{*}=\int_{0}^{Q^{*}} p t f(t) \mathrm{d} t+Q^{*} p\left\{1-F\left(Q^{*}\right)\right\}-Q^{*} w \\
\pi_{R}^{*}=\int_{0}^{Q^{*}} p t f(t) \mathrm{d} t+Q^{*} w-Q^{*} w \\
\pi_{R}^{*}=p \int_{0}^{Q^{*}} t f(t) \mathrm{d} t
\end{gathered}
$$

This can also be written as,

$$
\begin{gathered}
\pi_{R}^{*}=p \int_{0}^{Q^{*}} t f(t) \mathrm{d} t-Q^{*} p \int_{0}^{Q^{*}} f(t) \mathrm{d} t+Q^{*} p-Q^{*} w \\
\pi_{R}^{*}=p \int_{0}^{Q^{*}} t f(t) \mathrm{d} t-Q^{*} p F\left(Q^{*}\right)+Q^{*} p-Q^{*} w \\
\pi_{R}^{*}=Q^{*}(p-w)-p \int_{0}^{Q^{*}} F(t) \mathrm{d} t \\
\left\{\because \int_{0}^{Q^{*}} t f(t) \mathrm{d} t=Q^{*} F\left(Q^{*}\right)-\int_{0}^{Q^{*}} F(t) \mathrm{d} t, \text { Using Integration by parts }\right\} \\
\pi_{R}^{*}=p \int_{0}^{Q^{*}}\{1-F(t)\} \mathrm{d} t-Q^{*} w \\
\pi_{R}^{*}=p \int_{0}^{Q^{*}}\{1-P(D \leq t)\} \mathrm{d} t-Q^{*} w \\
\pi_{R}^{*}=p \int_{0}^{Q^{*}} P(D>t) \mathrm{d} t-Q^{*} w
\end{gathered}
$$

The variance of the profits when the optimal quantity is ordered is given by, 


$$
\begin{aligned}
& \operatorname{Var}\left(\pi_{R}^{*}\right)=p^{2}\left[\int_{0}^{Q^{*}} 2\left(Q^{*}-t\right) F(t) \mathrm{d} t-\left\{\int_{0}^{Q^{*}} F(t) \mathrm{d} t\right\}^{2}\right] \\
& =p^{2}\left[2 Q^{*} \int_{0}^{Q^{*}} F(t) \mathrm{d} t-2 \int_{0}^{Q^{*}} t F(t) \mathrm{d} t-\left\{\int_{0}^{Q^{*}} F(t) \mathrm{d} t\right\}^{2}\right] \\
& =p^{2}\left[\int_{0}^{Q^{*}} F(t) \mathrm{d} t\left\{2 Q^{*}-\left(\int_{0}^{Q^{*}} F(t) \mathrm{d} t\right)\right\}-2 \int_{0}^{Q^{*}} t F(t) \mathrm{d} t\right] \\
& =p^{2}\left[\left\{Q^{*} F\left(Q^{*}\right)-\int_{0}^{Q^{*}} t f(t) \mathrm{d} t\right\}\left\{2 Q^{*}-\left(Q^{*} F\left(Q^{*}\right)-\int_{0}^{Q^{*}} t f(t) \mathrm{d} t\right)\right\}-2 \int_{0}^{Q^{*}} t F(t) \mathrm{d} t\right] \\
& =p^{2}\left[\left\{Q^{*}\left\{1-\frac{w}{p}\right\}-\int_{0}^{Q^{*}} t f(t) \mathrm{d} t\right\}\left\{Q^{*}\left\{1+\frac{w}{p}\right\}+\int_{0}^{Q^{*}} t f(t) \mathrm{d} t\right\}-2 \int_{0}^{Q^{*}} t F(t) \mathrm{d} t\right] \\
& \operatorname{Var}\left(\pi_{R}^{*}\right)=p^{2}\left[\left(Q^{*}\right)^{2}\left\{1-\left(\frac{w}{p}\right)^{2}\right\}-\left(\int_{0}^{Q^{*}} t f(t) \mathrm{d} t\right)^{2}\right. \\
& \left.-2 Q^{*} \frac{w}{p} \int_{0}^{Q^{*}} t f(t) \mathrm{d} t-2 \int_{0}^{Q^{*}} t F(t) \mathrm{d} t\right]
\end{aligned}
$$

\section{Appendix C: Proof of Theorem 1}

Proof. Consider the expected profit function in the stochastic quantity ordered case,

$$
\begin{aligned}
& E\left[\pi_{R S}\right]=E_{Q, D}[\min (Q, D) p-Q w] \\
& =p \int_{0}^{\infty} \int_{0}^{\infty} \min (x, y) \hat{f}_{X, Y}(x, y) \mathrm{d} x \mathrm{~d} y-E[Q] w \\
& =p \int_{0}^{\infty} \int_{0}^{y} x g(x) \hat{f}(y) \mathrm{d} x \mathrm{~d} y+p \int_{0}^{\infty} \int_{y}^{\infty} y g(x) \hat{f}(y) \mathrm{d} x \mathrm{~d} y-E[Q] w \\
& =p \int_{0}^{\infty} \int_{x}^{\infty} x g(x) \hat{f}(y) \mathrm{d} y \mathrm{~d} x+p \int_{0}^{\infty} \int_{y}^{\infty} y g(x) \hat{f}(y) \mathrm{d} x \mathrm{~d} y-E[Q] w \\
& =p \int_{0}^{\infty} x g(x)\left\{\int_{x}^{\infty} \hat{f}(y) \mathrm{d} y\right\} \mathrm{d} x+p \int_{0}^{\infty} y \hat{f}(y)\left\{\int_{y}^{\infty} g(x) \mathrm{d} x\right\} \mathrm{d} y-E[Q] w \\
& =p \int_{0}^{\infty} x g(x)\left\{1-\int_{0}^{x} \hat{f}(y) \mathrm{d} y\right\} \mathrm{d} x+p \int_{0}^{\infty} y \hat{f}(y)\left\{1-\int_{0}^{y} g(x) \mathrm{d} x\right\} \mathrm{d} y-E[Q] w \\
& =p \int_{0}^{\infty} x g(x)\{1-\hat{F}(x)\} \mathrm{d} x+p \int_{0}^{\infty} y \hat{f}(y)\{1-G(y)\} \mathrm{d} y-E[Q] w \\
& =p \int_{0}^{\infty} x g(x) \mathrm{d} x-p \int_{0}^{\infty} x g(x) \hat{F}(x) \mathrm{d} x+p \int_{0}^{\infty} y \hat{f}(y) \mathrm{d} y-p \int_{0}^{\infty} y \hat{f}(y) G(y) \mathrm{d} y-E[Q] w \\
& =p \int_{0}^{\infty} x g(x) \mathrm{d} x+p \int_{0}^{\infty} y \hat{f}(y) \mathrm{d} y-p \int_{0}^{\infty} x g(x) \hat{F}(x) \mathrm{d} x-p \int_{0}^{\infty} y \hat{f}(y) G(y) \mathrm{d} y-E[Q] w
\end{aligned}
$$

We note that,

$$
\begin{aligned}
E[\max (X, Y)] & =\int_{0}^{\infty} \int_{0}^{\infty} \max (x, y) \hat{f}_{X, Y}(x, y) \mathrm{d} x \mathrm{~d} y \\
& =\int_{0}^{\infty} \int_{0}^{x} x g(x) \hat{f}(y) \mathrm{d} y \mathrm{~d} x+\int_{0}^{\infty} \int_{0}^{y} y g(x) \hat{f}(y) \mathrm{d} x \mathrm{~d} y \\
& =\int_{0}^{\infty} x g(x)\left\{\int_{0}^{x} \hat{f}(y) \mathrm{d} y\right\} \mathrm{d} x+\int_{0}^{\infty} y \hat{f}(y)\left\{\int_{0}^{y} g(x) \mathrm{d} x\right\} \mathrm{d} y \\
& =\int_{0}^{\infty} x g(x) \hat{F}(x) \mathrm{d} x+\int_{0}^{\infty} y \hat{f}(y) G(y) \mathrm{d} y
\end{aligned}
$$

This gives, 


$$
E\left[\pi_{R S}\right]=p E[Q]+p E[D]-p E[\max (Q, D)]-E[Q] w
$$

Alternately, noting that the original specification of the inventory problem has a $\min (Q, D)$ we can get the above by the following relationship between the minimum and maximum,

$$
\min (X, Y)+\max (X, Y)=X+Y
$$

For better outcomes under the stochastic quantity ordered scenario, we must have,

$$
\begin{gathered}
E\left[\pi_{R S}\right] \geq \hat{\pi}_{R}^{*} \\
p E[Q]+p E[D]-p E[\max (Q, D)]-E[Q] w \geq p \int_{0}^{\hat{Q}^{*}} t \hat{f}(t) \mathrm{d} t \\
E[Q]\left\{1-\frac{w}{p}\right\}+E[D]-E[\max (Q, D)] \geq \int_{0}^{\hat{Q}^{*}} t \hat{f}(t) \mathrm{d} t
\end{gathered}
$$

\section{Appendix D: Proof of Proposition 1}

Proof. From the criteria in Theorem 1, the result is immediate

$$
\begin{gathered}
E\left[\pi_{R S}\right] \geq \hat{\pi}_{R}^{*} \\
E_{Q, D}[\min (Q, D) p-Q w] \geq E_{D}\left[\min \left(\hat{Q}^{*}, D\right) p-\hat{Q}^{*} w\right] \\
E_{Q, D}[Q+D-\max (Q, D) p-Q w] \geq E_{D}\left[\hat{Q}^{*}+D-\max \left(\hat{Q}^{*}, D\right) p-\hat{Q}^{*} w\right] \\
E_{Q, D}[\max (Q, D)] \leq E_{D}\left[\max \left(\hat{Q}^{*}, D\right)\right] \\
\int_{0}^{\infty} x g(x) \hat{F}(x) \mathrm{d} x+\int_{0}^{\infty} y \hat{f}(y) G(y) \mathrm{d} y \leq \int_{0}^{\hat{Q}^{*}} \hat{Q}^{*} \hat{f}(t) \mathrm{d} t+\int_{\hat{Q}^{*}}^{\infty} t \hat{f}(t) \mathrm{d} t \\
\int_{0}^{\infty} x g(x) \hat{F}(x) \mathrm{d} x+\int_{0}^{\infty} y \hat{f}(y) G(y) \mathrm{d} y \leq \hat{Q}^{*} \hat{F}\left(\hat{Q}^{*}\right)+\int_{Q^{*}}^{\infty} t \hat{f}(t) \mathrm{d} t
\end{gathered}
$$

Submit or recommend next manuscript to SCIRP and we will provide best service for you:

Accepting pre-submission inquiries through Email, Facebook, LinkedIn, Twitter, etc. A wide selection of journals (inclusive of 9 subjects, more than 200 journals)

Providing 24-hour high-quality service

User-friendly online submission system

Fair and swift peer-review system

Efficient typesetting and proofreading procedure

Display of the result of downloads and visits, as well as the number of cited articles

Maximum dissemination of your research work

Submit your manuscript at: http://papersubmission.scirp.org/

Or contact tel@scirp.org 